Supplement of Hydrol. Earth Syst. Sci., 24, 2999-3014, 2020

https://doi.org/10.5194/hess-24-2999-2020-supplement

(c) Author(s) 2020. This work is distributed under

the Creative Commons Attribution 4.0 License.

(c) (1)
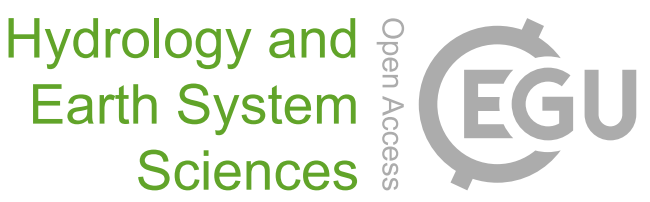

Supplement of

\title{
Partitioning growing season water balance within a forested boreal catchment using sap flux, eddy covariance, and a process-based model
}

Nataliia Kozii et al.

Correspondence to: Niles Hasselquist (niles.hasselquist@gmail.com)

The copyright of individual parts of the supplement might differ from the CC BY 4.0 License. 


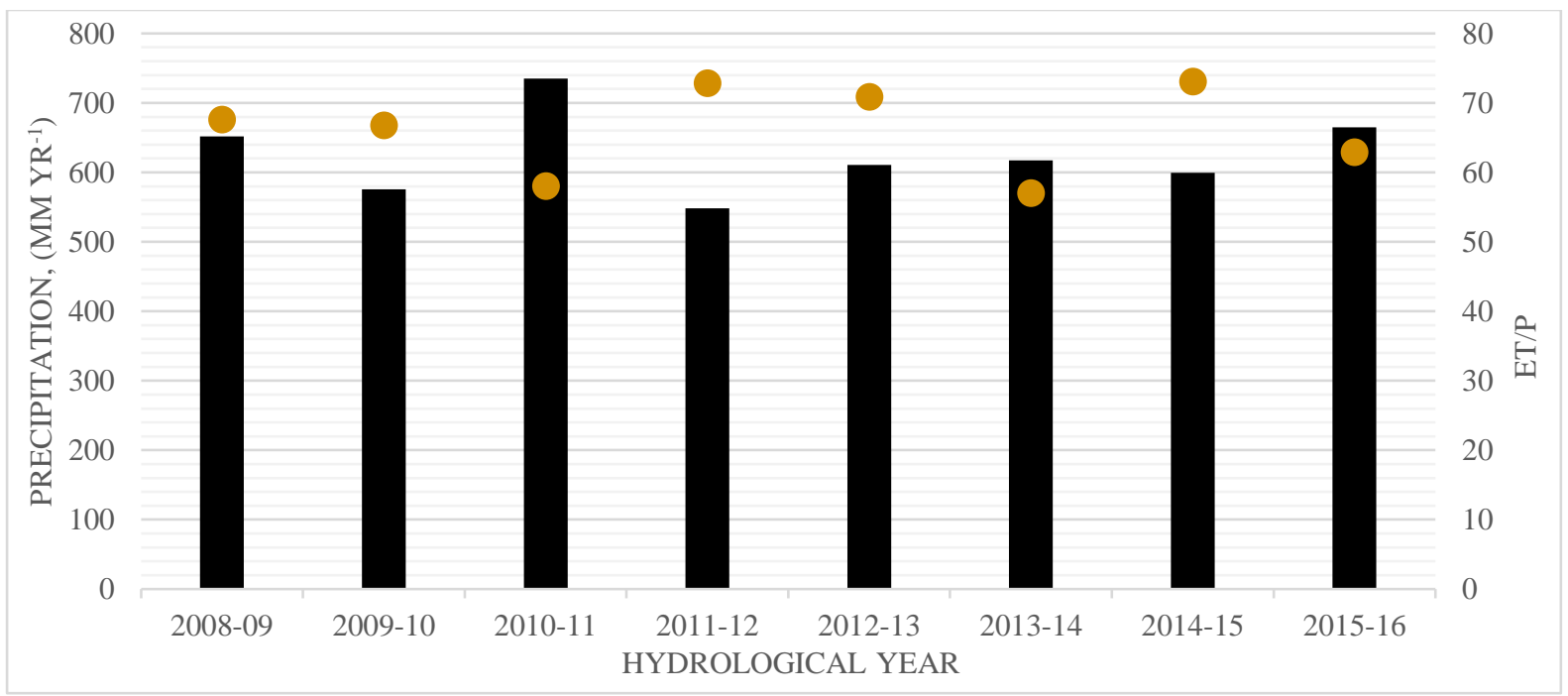

Figure S1. Annual precipitation (dots) and fraction of ET/P (bars) over 8 hydrological years within the $\mathrm{C} 2$ catchment. 
Table S1. The 10 canopy attributes that has the highest correlation to seasonal $\mathrm{I}_{C} / \mathrm{P}$.

\begin{tabular}{|c|c|c|c|c|c|c|c|c|c|c|c|}
\hline \multirow[b]{2}{*}{$\begin{array}{l}\text { Throughfall } \\
\text { collector }\end{array}$} & \multirow[b]{2}{*}{$\begin{array}{l}\text { Seasonal } \\
\mathrm{I}_{\mathrm{C}} / \mathrm{P}\end{array}$} & \multicolumn{10}{|c|}{ Canopy Attributes } \\
\hline & & $\begin{array}{l}\text { ElevMAD } \\
\text { medium }\end{array}$ & EleviQ & $\begin{array}{l}\text { Total return } \\
\text { count }\end{array}$ & $\begin{array}{c}\text { Elev } \\
\text { variance }\end{array}$ & ElevL2 & $\begin{array}{c}\text { Total return } \\
\text { Count above } 200\end{array}$ & $\begin{array}{l}\text { All returns } \\
\text { Above } 200\end{array}$ & ElevAAD & $\begin{array}{l}\text { Totall } \\
\text { returns }\end{array}$ & Elevstddev \\
\hline 1 & 0.30 & 2.9 & 6.5 & 260.0 & 29.0 & 2.9 & 213.0 & 213.0 & 4.4 & 260.0 & 5.4 \\
\hline 2 & 0.23 & 2.7 & 4.5 & 167.0 & 16.9 & 2.3 & 150.0 & 150.0 & 3.1 & 167.0 & 4.1 \\
\hline 3 & 0.40 & 2.8 & 7.1 & 260.0 & 22.1 & 2.6 & 212.0 & 212.0 & 3.9 & 260.0 & 4.7 \\
\hline 4 & 0.76 & 5.1 & 10.5 & 339.0 & 39.8 & 3.6 & 298.0 & 298.0 & 5.4 & 339.0 & 6.3 \\
\hline 5 & 0.59 & 4.0 & 8.4 & 271.0 & 28.3 & 3.1 & 216.0 & 216.0 & 4.5 & 271.0 & 5.3 \\
\hline 6 & 0.48 & 3.8 & 10.0 & 257.0 & 34.1 & 3.3 & 216.0 & 216.0 & 5.0 & 257.0 & 5.8 \\
\hline 7 & 0.33 & 3.3 & 5.9 & 187.0 & 21.4 & 2.6 & 122.0 & 122.0 & 3.7 & 187.0 & 4.6 \\
\hline 8 & 0.69 & 4.9 & 9.2 & 298.0 & 29.9 & 3.2 & 252.0 & 252.0 & 4.7 & 298.0 & 5.5 \\
\hline 9 & 0.21 & 3.3 & 8.3 & 231.0 & 27.8 & 3.0 & 200.0 & 200.0 & 4.5 & 231.0 & 5.3 \\
\hline 10 & 0.30 & 1.7 & 3.2 & 263.0 & 14.0 & 2.0 & 229.0 & 229.0 & 2.8 & 263.0 & 3.7 \\
\hline 11 & 0.20 & 2.3 & 8.0 & 207.0 & 26.3 & 2.8 & 164.0 & 164.0 & 4.3 & 207.0 & 5.1 \\
\hline 12 & 0.18 & 1.9 & 4.4 & 256.0 & 9.2 & 1.7 & 236.0 & 236.0 & 2.5 & 256.0 & 3.0 \\
\hline 13 & 0.27 & 2.1 & 3.8 & 219.0 & 6.5 & 1.5 & 169.0 & 169.0 & 2.2 & 219.0 & 2.6 \\
\hline 14 & 0.29 & 2.5 & 4.8 & 249.0 & 10.0 & 1.8 & 207.0 & 207.0 & 2.7 & 249.0 & 3.2 \\
\hline 15 & 0.10 & 1.6 & 3.1 & 190.0 & 6.8 & 1.5 & 126.0 & 126.0 & 2.1 & 190.0 & 2.6 \\
\hline 16 & 0.26 & 2.3 & 4.6 & 223.0 & 9.0 & 1.7 & 193.0 & 193.0 & 2.4 & 223.0 & 3.0 \\
\hline 17 & 0.30 & 2.8 & 5.5 & 140.0 & 12.3 & 2.0 & 132.0 & 132.0 & 3.0 & 140.0 & 3.5 \\
\hline 18 & 0.02 & 0.0 & 0.0 & 101.0 & 0.0 & 0.0 & 0.0 & 0.0 & 0.0 & 0.0 & 0.0 \\
\hline 19 & 0.37 & 3.4 & 6.6 & 221.0 & 19.6 & 2.5 & 206.0 & 206.0 & 3.7 & 221.0 & 4.4 \\
\hline 20 & 0.30 & 2.1 & 4.0 & 179.0 & 17.9 & 2.2 & 156.0 & 156.0 & 3.1 & 179.0 & 4.2 \\
\hline 21 & 0.07 & $\begin{array}{l}2.1 \\
1.1\end{array}$ & 2.2 & 112.0 & 3.7 & $\begin{array}{l}2.2 \\
1.0\end{array}$ & 42.0 & 42.0 & $\begin{array}{l}5.1 \\
1.3\end{array}$ & 112.0 & 1.9 \\
\hline 22 & 0.25 & 1.2 & 2.6 & 184.0 & 2.6 & 0.9 & 138.0 & 138.0 & 1.4 & 184.0 & 1.6 \\
\hline 23 & 0.17 & 1.1 & 2.3 & 172.0 & 3.3 & 1.0 & 128.0 & 128.0 & 1.4 & 172.0 & 1.8 \\
\hline 24 & 0.15 & 1.1 & 2.2 & 204.0 & 2.3 & 0.9 & 133.0 & 133.0 & 1.2 & 204.0 & 1.5 \\
\hline 25 & 0.27 & 1.0 & 1.6 & 232.0 & 1.8 & 0.8 & 154.0 & 154.0 & 1.0 & 232.0 & 1.3 \\
\hline \multicolumn{2}{|c|}{$\begin{array}{l}\text { Correlation between IC/P } \\
\text { and canopy attributes }\end{array}$} & 0.77 & 0.62 & 0.60 & 0.59 & 0.59 & 0.57 & 0.57 & 0.57 & 0.55 & 0.54 \\
\hline
\end{tabular}

ElevMADmedium $=$ the median of the absolute deviations from the overall median

ElevIQ = interquartile distance of elevations within the plot

Total return count $=$ total number of all returns of the plot

Elevvariance $=$ variance of elevations within the plot

ElevL2 = second L-moment of the return heights of the plot

Total return count above $200=$ total number of all returns of the plot above 2 meters 
All returns above $200=$ number of returns above 2 meters

ElevAAD = average absolute deviation

Totall returns $=$ number of all returns of the plot

Elevstddev $=$ standard deviation of elevations within the plot 


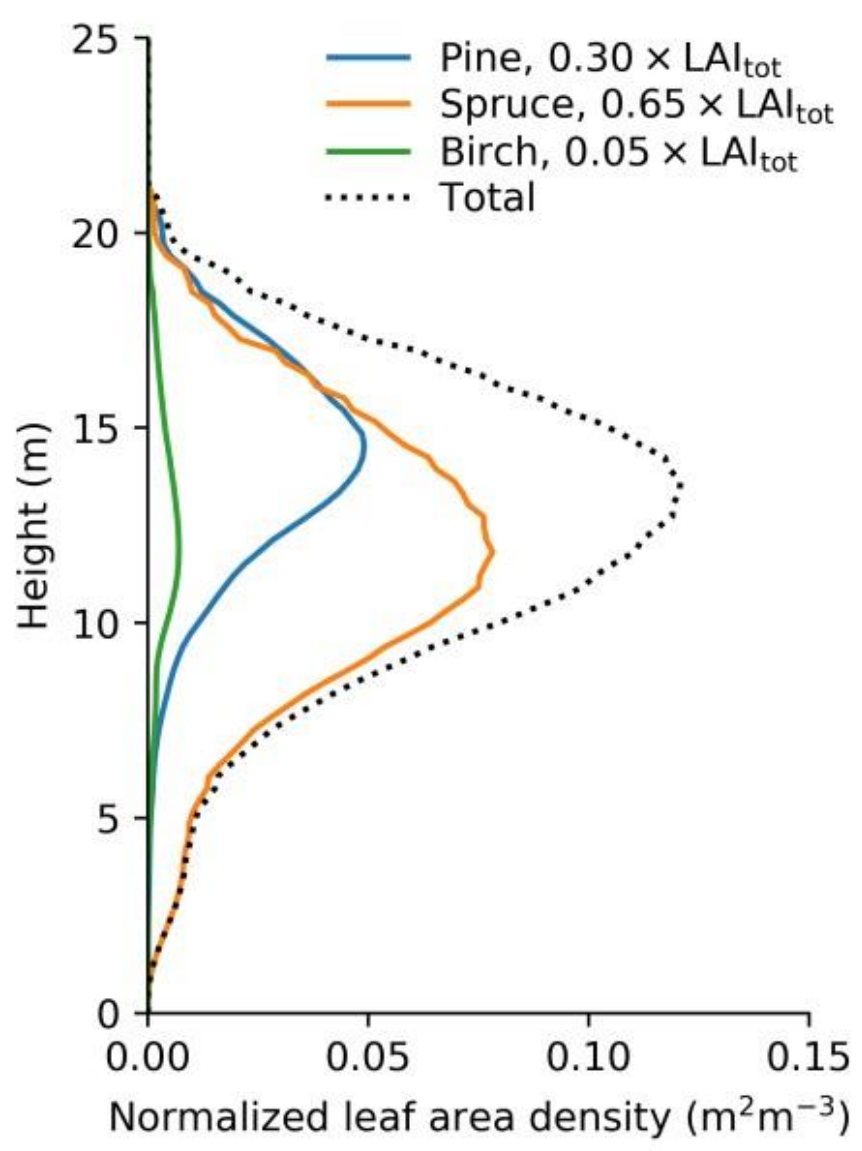

Figure S2. Normalized leaf area density distributions for main tree species and their fraction of total stand leaf area index ( $\left.\mathrm{LAI}_{\text {tot }}\right)$ with the $\mathrm{C} 2$ subcatchment. 
Table S2. Model parameter values applied in simulations (for plant type specific parameters see Table S2).

\begin{tabular}{lll}
\hline Description & Parameter value & Source \\
\hline Canopy radiation parameters & & \\
Clumping coefficient (-) & 0.7 & (Campbell and Norman, 1998) \\
Leaf-angle distribution (-) & 1.0 (spherical) & (Campbell and Norman, 1998) \\
Shoot PAR albedo (-) & 0.1 & Adjusted to match canopy albedo \\
Shoot NIR albedo (-) & 0.39 & Adjusted to match canopy albedo \\
Leaf emissivity (-) & 0.98 & (Campbell and Norman, 1998) \\
\hline
\end{tabular}

Canopy flow parameters

Normalized horizontal pressure gradient $\left(\mathrm{m} \mathrm{s}^{-2}\right) \quad 0.01$

Foliage drag coefficient (-) $0.15 \quad$ (Lee et al., 1994)

Schmidt number for $\mathrm{H}_{2} \mathrm{O}, \mathrm{T}, \mathrm{CO}_{2}$

\begin{tabular}{lll}
\hline Canopy interception parameters & & \\
Maximum interception storage (mm) & $0.2-0.5 \times$ LAI & (Watanabe and Mizutani, 1996) \\
Leaf orientation factor (-) & 0.5 (random) & (Watanabe and Mizutani, 1996)
\end{tabular}

\begin{tabular}{|c|c|c|}
\hline \multicolumn{3}{|c|}{ Plant type parameters for photosynthesis-stomatal conductance model } \\
\hline Carboxylation capacity $\mathrm{V}_{\mathrm{cmax}}$ at $25^{\circ} \mathrm{C}\left(\mu \mathrm{mol} \mathrm{m} \mathrm{m}^{-2} \mathrm{~s}^{-1}\right)^{\mathrm{a}}$ & $\mathrm{V}_{\mathrm{cmax} 25}$ & see Table 2 \\
\hline Electron transport capacity $\mathrm{J}_{\max }$ at $25^{\circ} \mathrm{C}\left(\mu \mathrm{mol} \mathrm{m} \mathrm{m}^{-2} \mathrm{~s}^{-1}\right.$ & $1.97 \times \mathrm{V}_{\mathrm{cmax} 25}$ & (Kattge and Knorr, 2007) \\
\hline Leaf dark respiration rate $\mathrm{r}_{\mathrm{d}}$ at $25^{\circ} \mathrm{C}\left(\mu \mathrm{mol} \mathrm{m} \mathrm{m}^{-2} \mathrm{~s}^{-1}\right)^{\mathrm{a}}$ & $0.023 \times \mathrm{V}_{\mathrm{cmax} 25}$ & (Launiainen et al., 2015) \\
\hline Co-limitation parameter & 0.95 & \\
\hline Curvature of electron transport light response (-) & 0.7 & (Launiainen et al., 2015) \\
\hline Quantum yield parameter $\left(\mathrm{mol} \mathrm{mol}^{-1}\right)$ & 0.2 & (Launiainen et al., 2015) \\
\hline Stomatal model slope $(-)^{\mathrm{b}}$ & $\mathrm{g}_{1}$ & see Table 2 \\
\hline Residual conductance $\left(\mathrm{mol} \mathrm{m}^{-2} \mathrm{~s}^{-1}\right)^{\mathrm{b}}$ & $\mathrm{g}_{0}$ & see Table 2 \\
\hline \multicolumn{3}{|l|}{ Bryophyte layer parameters } \\
\hline Height (m) & 0.095 & (Soudzilovskaia et al., 2013) \\
\hline Roughness height (m) & 0.01 & (Launiainen et al., 2015) \\
\hline Bulk density $\left(\mathrm{kg} \mathrm{m}^{-3}\right)$ & 17.1 & (Soudzilovskaia et al., 2013) \\
\hline Total pore volume $\left(\mathrm{m}^{3} \mathrm{~m}^{-3}\right)$ & 0.98 & (Voortman et al., 2014) \\
\hline Maximum gravimetric water content, $\mathrm{w}_{\max }\left(\mathrm{g} \mathrm{g}^{-1}\right)$ & 10 & (Soudzilovskaia et al., 2013) \\
\hline Minimum gravimetric water content $\left(\mathrm{g} \mathrm{g}^{-1}\right)$ & 1.5 & (Launiainen et al., 2015) \\
\hline Van Genuchten water retention parameter $\left(\mathrm{cm}^{-1}\right)$ & 0.13 & (Voortman et al., 2014) \\
\hline Van Genuchten water retention parameter (-) & 2.17 & (Voortman et al., 2014) \\
\hline Saturated hydraulic conductivity $(\mathrm{m} / \mathrm{h})$ & $4.2 \times 10^{-4}$ & (Voortman et al., 2014) \\
\hline
\end{tabular}


Pore connectivity (-)

Emissivity (-)

PAR albedo at $\mathrm{w}_{\max }(-)^{\mathrm{c}}$

NIR albedo at $\mathrm{w}_{\max }(-)^{\mathrm{c}}$
Depth (m)

Porosity $\left(\mathrm{m}^{3} / \mathrm{m}^{3}\right)$

Residual water content $\left(\mathrm{m}^{3} / \mathrm{m}^{3}\right)$

Van Genuchten water retention parameter $\left(\mathrm{cm}^{-1}\right)$

Van Genuchten water retention parameter (-)

Saturated hydraulic conductivity $(\mathrm{m} / \mathrm{h})$

Thermal conductivity of solids $(\mathrm{W} / \mathrm{m} / \mathrm{K})$

Heat capacity of solids $\left(\mathrm{MJ} / \mathrm{m}^{3} / \mathrm{K}\right)$
0.8

0.01

0.7

1.25

0.015

2.17

2.35
(Voortman et al., 2014)

(Campbell and Norman, 1998)

(Bubier et al., 1997)

(Bubier et al., 1997)

$2 \times$ measurement depth of soil temperature and moisture

(Launiainen et al., 2015)

(Launiainen et al., 2015)

(Launiainen et al., 2015)

(Launiainen et al., 2015)

(Launiainen et al., 2015)

Derived from soil composition ${ }^{\mathrm{d}}$

Derived from soil composition ${ }^{\mathrm{d}}$

\footnotetext{
${ }^{a}$ Temperature response curves of $\mathrm{V}_{\mathrm{cmax}}$ and $\mathrm{J}_{\max }$ are adopted from Kattge and Knorr (2007) and of $\mathrm{r}_{\mathrm{d}}$ from Launiainen et al. (2015)

${ }^{b}$ Parameters of Medlyn et al. (2011) optimal stomatal conductance model

${ }^{\mathrm{c}}$ Response of albedo to bryophyte water content (Kieloaho and Launianen, 2018)

${ }^{\mathrm{d}}$ Soil composition of top soil layer adopted from Jauhiainen (2004) and thermal properties soil materials from Tian et al. (2016) and Campbell and Norman (1998)
} 
Table S3. Plant type specific model parameter values applied in simulations.

\begin{tabular}{|c|c|c|c|c|}
\hline \multirow[t]{2}{*}{ Description } & \multicolumn{4}{|c|}{ Parameter value } \\
\hline & Spruce & Pine & Birch & Understory \\
\hline Maximum LAI, $\mathrm{LAI}_{\max }\left(\mathrm{m}^{2} / \mathrm{m}^{2}\right)^{\mathrm{a}}$ & $0.31 \times \mathrm{LAI}_{\text {tot }}$ & $0.64 \times \mathrm{LAI}_{\mathrm{tot}}$ & $0.05 \times \mathrm{LAI}_{\mathrm{tot}}$ & $0.4-0.8$ \\
\hline Minimum LAI $\left(\mathrm{m}^{2} / \mathrm{m}^{2}\right)^{\mathrm{a}}$ & $0.8 \times \mathrm{LAI}_{\max }$ & $0.8 \times \mathrm{LAI}_{\max }$ & $0.1 \times \mathrm{LAI}_{\max }$ & $0.5 \times \mathrm{LAI}_{\max }$ \\
\hline Minimum value for seasonal cycle modifier $(-)^{b}$ & 0.1 & 0.1 & 0.01 & 0.01 \\
\hline Characteristic leaf length scale (m) & 0.02 & 0.02 & 0.05 & 0.05 \\
\hline Nitrogen attenuation coefficient (-) & 0.5 & 0.5 & 0.2 & 0 \\
\hline $\mathrm{V}_{\mathrm{cmax} 25}\left(\mu \mathrm{mol} \mathrm{m} \mathrm{m}^{-2} \mathrm{~s}^{-1}\right)$ & 60 & 50 & 45 & 40 \\
\hline Stomatal model slope $g_{1}(-)$ & 2.5 & 2.5 & 4.5 & 4.5 \\
\hline Residual conductance $\mathrm{g}_{0}\left(\mathrm{~mol} \mathrm{~m} \mathrm{~m}^{-2} \mathrm{~s}^{-1}\right)$ & 0.004 & 0.004 & 0.01 & 0.01 \\
\hline
\end{tabular}

${ }^{\mathrm{a}}$ Seasonal development of LAI starts when the degree day sum $\left(\mathrm{T}_{\text {base }}=5^{\circ} \mathrm{C}\right)$ exceeds 45 days and reaches maturation at 250 days. Leaf senescence in autumn follows Launiainen et al. (2015). $\mathrm{LAI}_{\mathrm{tot}}=3.4-6.9 \mathrm{~m}^{2} \mathrm{~m}^{-2}$.

${ }^{\mathrm{b}}$ Seasonal cycle modifier for photosynthetic capacity is based on the delayed effect of temperature (Kolari et al., 2007; Launiainen et al., 2015). 


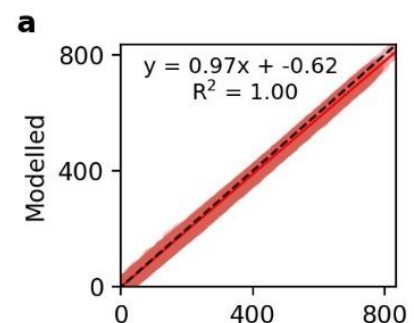

b

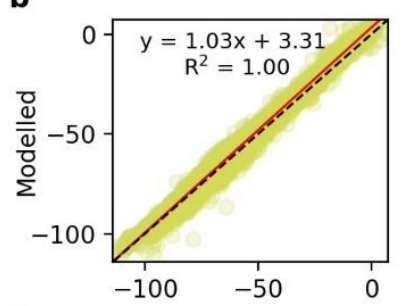

c

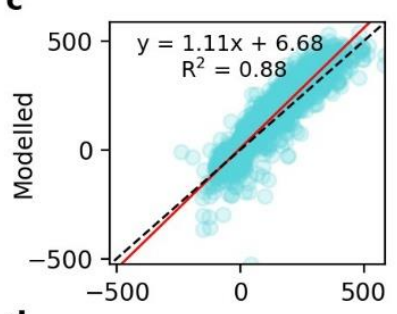

d

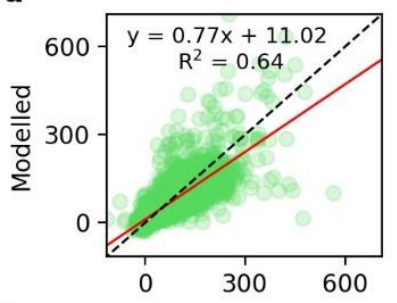

e

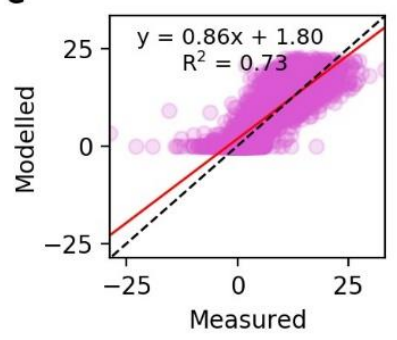

Net shortwave radiation [ $\mathrm{W} \mathrm{m}^{-2}$ ]
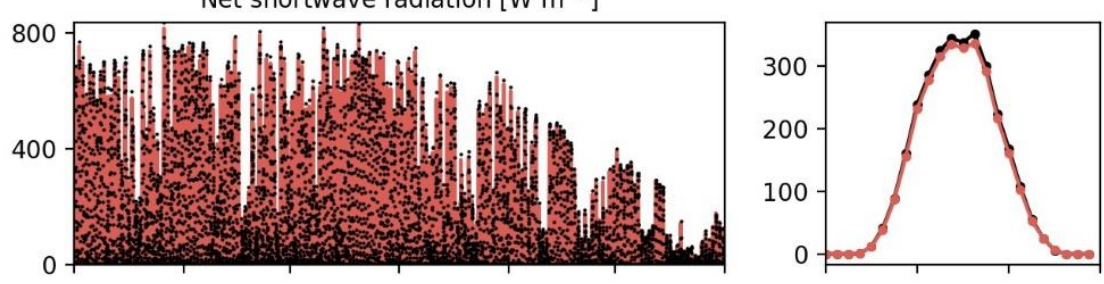

Net longwave radiation $\left[\mathrm{W} \mathrm{m}^{-2}\right]$
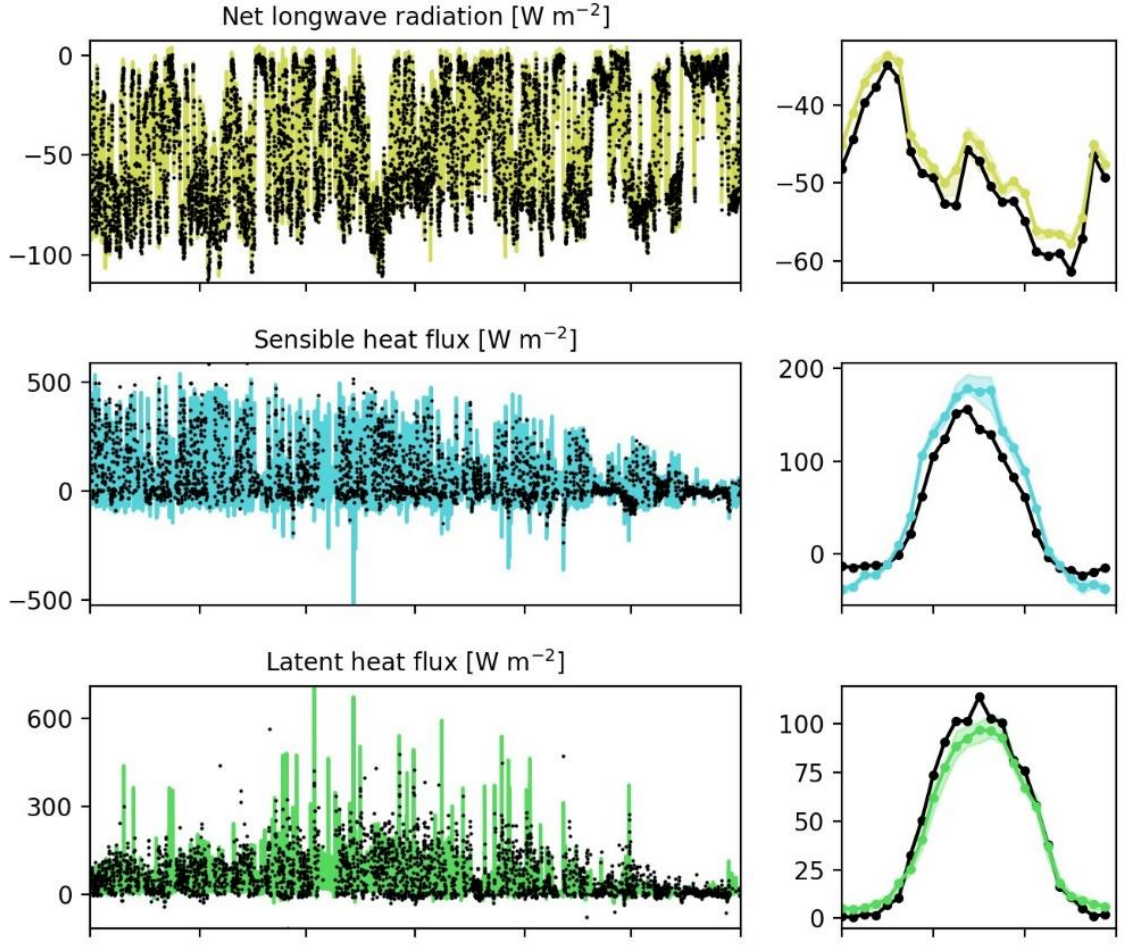

Gross primary production $\left[\mu \mathrm{mol} \mathrm{m} \mathrm{m}^{-2} \mathrm{~s}^{-1}\right]$
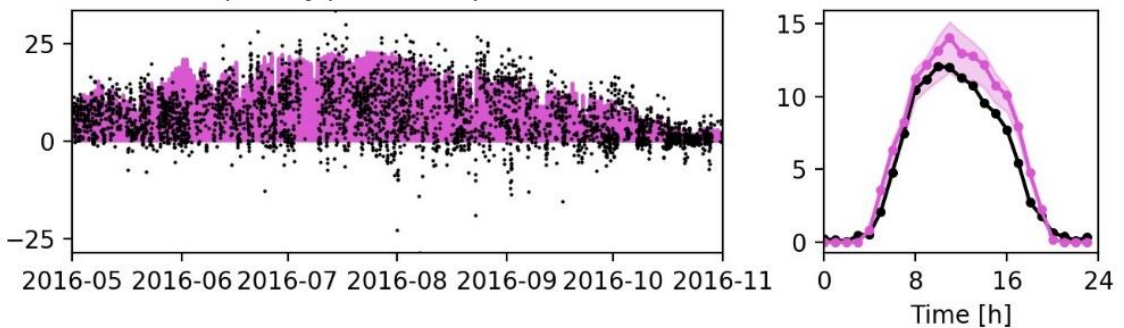

Figure S3. Scatter plot comparison, time series and average diurnal cycle for measured and modelled components of net radiation (a-b), sensible heat flux (c), latent heat flux (d), and gross primary production (e). Eddy covariance data (c-e) is non-gapfilled and only model results of times with data available are plotted in diurnal cycles. Modeled data are in colors and measured in black. 


\section{References}

Bubier, J.L., Rock, B.N., Crill, P.M., 1997. Spectral reflectance measurements of boreal wetland and forest mosses. Journal of Geophysical Research: Atmospheres 102, 29483 29494. https://doi.org/10.1029/97JD02316

Campbell, G.S., Norman, J.M., 1998. Introduction to Environmental Biophysics, 2nd ed. Springer.

Jauhiainen, M., 2004. Relationships of particle size distribution curve, soil water retention curve and unsaturated hydraulic conductivity and their implications on water balance of forested and agricultural hillslopes. Helsinki University of Technology.

Kattge, J., Knorr, W., 2007. Temperature acclimation in a biochemical model of photosynthesis: a reanalysis of data from 36 species. Plant Cell Environ. 30, 1176-1190. https://doi.org/10.1111/j.1365-3040.2007.01690.x

Kieloaho, A.-J., Launianen, S., 2018. Effects of functional traits of bryophyte layer on water cycling and energy balance in boreal and arctic ecosystems, in: EGU General Assembly Conference Abstracts. p. 11786.

Kolari, P., Lappalainen, H.K., Hänninen, H., Hari, P., 2007. Relationship between temperature and the seasonal course of photosynthesis in Scots pine at northern timberline and in southern boreal zone. Tellus Series B-Chemical and Physical Meteorology 59, 542552.

Launiainen, S., Katul, G.G., Lauren, A., Kolari, P., 2015. Coupling boreal forest CO2, H2O and energy flows by a vertically structured forest canopy - Soil model with separate bryophyte layer. Ecological Modelling 312, 385-405.

https://doi.org/10.1016/j.ecolmodel.2015.06.007

Lee, X., Shaw, R.H., Black, T.A., 1994. Modelling the effect of mean pressure gradient on the mean flow within forests. Agricultural and Forest Meteorology 68, 201-212. https://doi.org/10.1016/0168-1923(94)90036-1

Medlyn, B.E., Duursma, R.A., Eamus, D., Ellsworth, D.S., Prentice, I.C., Barton, C.V.M., Crous, K.Y., Angelis, P.D., Freeman, M., Wingate, L., 2011. Reconciling the optimal and empirical approaches to modelling stomatal conductance. Global Change Biology 17, 21342144. https://doi.org/10.1111/j.1365-2486.2010.02375.x

Soudzilovskaia, N.A., van Bodegom, P.M., Cornelissen, J.H., 2013. Dominant bryophyte control over high-latitude soil temperature fluctuations predicted by heat transfer traits, field moisture regime and laws of thermal insulation. Functional Ecology 27, 1442-1454. 
Stenberg, P., 1996. Correcting LAI-2000 estimates for the clumping of needles in shoots of conifers. Agricultural and Forest Meteorology 79, 1-8. https://doi.org/10.1016/01681923(95)02274-0

Tian, Z., Lu, Y., Horton, R., Ren, T., 2016. A simplified de Vries-based model to estimate thermal conductivity of unfrozen and frozen soil. European Journal of Soil Science 67, 564572. https://doi.org/10.1111/ejss.12366

Voortman, B.R., Bartholomeus, R.P., Bodegom, P.M. van, Gooren, H., Zee, S.E.A.T.M. van der, Witte, J.-P.M., 2014. Unsaturated hydraulic properties of xerophilous mosses: towards implementation of moss covered soils in hydrological models. Hydrological Processes 28, 6251-6264. https://doi.org/10.1002/hyp.10111

Watanabe, T., Mizutani, K., 1996. Model study on micrometeorological aspects of rainfall interception over an evergreen broad-leaved forest. Agricultural and Forest Meteorology 80, 195-214. https://doi.org/10.1016/0168-1923(95)02301-1 